\title{
Electric brain responses reveal gender differences in music processing
}

\author{
Stefan Koelsch, ${ }^{1,2, C A}$ Burkhard Maess, ${ }^{2}$ Tobias Grossmann ${ }^{2}$ and Angela D. Friederici ${ }^{2}$ \\ 'Harvard Medical School, Boston, USA; ${ }^{2}$ Max Planck Institute of Cognitive Neuroscience, Stephan Str. la, 04103 Leipzig, Germany \\ CA,2 Corresponding Author and Address: koelsch@cns.mpg.de \\ Received 25 January 2003; accepted 6 February 2003 \\ DOI: 10.1097/0l.wnr.0000065762.60383.67
}

\begin{abstract}
The present study investigates gender differences in the functional organization of the brain for music processing. In the language domain, males appear to have greater left hemisphere control than females. Despite some overlap of neural structures and processes for the perception of music and language, gender differences of musical functions have so far not been reported. Data sets of three previous music experiments with event-related brain potentials (ERPs) were pooled and re-analyzed. Results demonstrate that
\end{abstract}

an electrophysiological correlate of music-syntactic processing (ERAN, or music-syntactic MMN) is generated bilaterally in females, and with right hemispheric predominance in males. The present findings indicate that gender differences for the analysis of auditory information are not restricted to processes in the linguistic domain such as syntax, semantics, and phonology. NeuroReport 14:709-713 (c) 2003 Lippincott Williams \& Wilkins.

Key words: Auditory processing; EEG; ERAN; ERP; Gender differences; MMN; Music; N5

\section{INTRODUCTION}

The brains of females and males have been reported to differ both structurally and functionally [1]. For the processing of language, brain imaging studies indicate gender differences in both anterior (inferior frontal gyrus) [2,3] and posterior (temporal) [4,5] language areas, activations of language functions being more preponderant in the left hemisphere in males, and more bilateral in females. These findings concur with lesion studies reporting that the incidence of aphasia is considerably higher in males than females with left-hemisphere lesions [6], supporting the notion of greater left hemisphere control of verbal functions in men than in women [1]. Similar gender differences have been reported for children in dichotic listening paradigms [7], and the prevalence of several language disorders in boys relative to girls has been taken to reflect the greater asymmetry of language functions in the male brain $[1,6,8]$.

Despite considerable overlap of neural structures and processes underlying the perception of language and music [9-15], gender differences for the processing of music have so far not been reported. In order to investigate gender differences in regard to music processing, we reanalyzed the data sets of three recent experiments [15,16]. In these experiments, music processing was investigated using EEG and event-related brain potentials (ERPs). Chord sequences were presented to participants who did not have formal musical training (non-musicians). Each sequence consisted of five chords, and sequences were composed in a way that a musical context was built up towards the end of each sequence. Infrequently, an inappropriate harmony was presented at either the third or the fifth position of a chord sequence (Fig. 1). These inappropriate harmonies elicit a relatively early electric brain response with negative polarity, an amplitude maximum around $200 \mathrm{~ms}$, and a right anterior scalp distribution. As a working term, this effect was labelled the early right anterior negativity (ERAN) [15].

The ERAN is taken to reflect the violation of a musical sound expectancy; the generation of these expectancies is based on (implicit) knowledge of complex musical regularities: in the mentioned experiments regularities of majorminor tonal music (e.g. classical Western European music) which are described by music theory. Deviant acoustic events that are not presented within a complex musical context do not elicit an ERAN [16]. The ERAN can be elicited pre-attentively [17], suggesting that musical expectancies are automatically generated during the perception of a musical context. Moreover, the ERAN is larger in subjects with explicit musical long-term training (musicians) than in non-musicians [18], reflecting that a more precise representation of musical regularities leads to more specific musical expectancies. The neural generators of the ERAN have been localized in the inferior fronto-lateral cortex (inferior part of the pars opercularis, Brodmann's area 44, in the left hemisphere often denoted as Broca's area) [14]. The ERAN is usually followed by a negative potential around $500 \mathrm{~ms}$ (denoted N5) $[15,17,18]$, which is taken to reflect processes of harmonic integration. The locations of the neural 


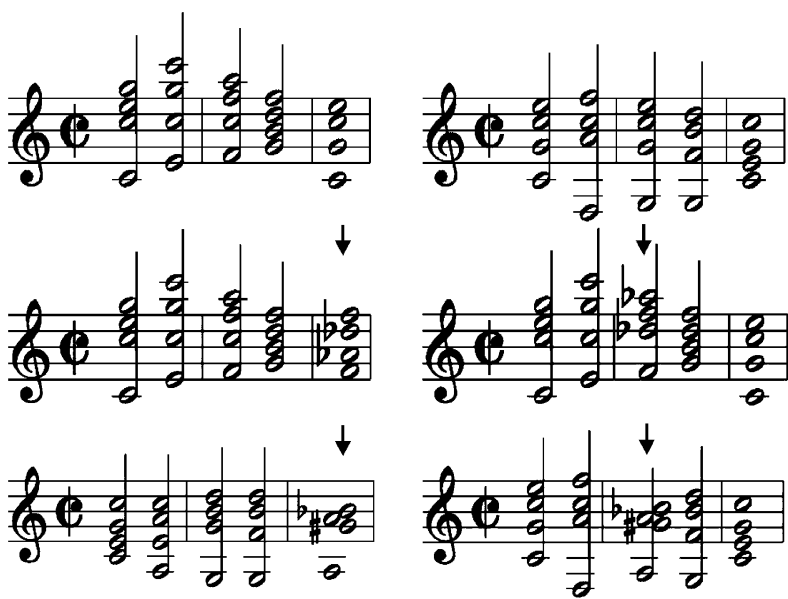

Fig. I. Examples of chord sequences consisting of in-key chords only (top row), containing a Neapolitan chord at the third and fifth position (middle row), and containing clusters at the third and fifth position (bottom row). The arrows indicate Neapolitans and clusters.

generators of the N5 are not yet clear; a recent fMRI study indicates that, in addition to fronto-lateral, superior temporal areas are activated during the processing of unexpected musical events [19]. The aim of the present study was to determine whether ERAN and N5 differ between males and females. In order to increase the signal-to-noise ratio of the data, we pooled 62 data sets from three previous EEG experiments in which ERAN and N5 were elicited.

\section{MATERIALS AND METHODS}

Subjects: Data sets from 62 right-handed subjects (age 1931 years, 31 females) were analyzed: the data sets from Experiment $1(n=18)$ and Experiment $2(n=18)$ of Koelsch et al. [15], and the 26 data sets from Block 2 of Koelsch et al. [16]. All subjects were non-musicians with no formal musical training (apart from normal school education).

Stimuli: In Experiment 1 [15], 172 chord sequences were presented, each consisting of five chords. The first chord was the tonic of the following cadence, chords at the second position were: tonic, mediant, submediant, subdominant, dominant to the dominant, secondary dominant to mediant, secondary dominant to submediant, secondary dominant to supertonic; at the third position: subdominant, dominant, dominant six-four chord, Neapolitan sixth chord; at the fourth position: dominant seventh chord; at the fifth position: tonic or Neapolitan sixth chord. In $25 \%$ of the sequences, a Neapolitan chord was presented at the third position, and in another $25 \%$ a Neapolitan was presented at the fifth position of a chord sequence. Sequences with Neapolitan chords and in-key sequences were randomly intermixed. Each variation of a Neapolitan chord was presented at the third position in one sequence, and at the fifth position in another. Presentation time of chords 1-4 was $600 \mathrm{~ms}$, chord 5 was presented for $1200 \mathrm{~ms}$. All chords had the same decay of loudness, i.e. Neapolitan chords at the third and at the fifth position were on average physically identical (within the first $600 \mathrm{~ms}$ ). Chords were played under computerized control via MIDI on a synthesizer. In $10 \%$ of the sequences, an in-key chord at the second, third, fourth or fifth position was played by another instrument than piano (e.g. harpsichord, celesta, marimba).

In Experiment 2 [15], stimuli were the same as in Experiment 1, except that Neapolitan chords were replaced by half-tone clusters. Clusters represent a higher degree of violation than Neapolitans; consequently, ERAN and N5 elicited by clusters had a larger amplitude than when elicited by Neapolitans (for details see [15]).

In Block 2 [16], stimuli were the same as in Experiment 1, except that (a) 255 sequences were presented, (b) no secondary dominants were presented at the second position, (c) no deviant instruments were presented, and (d) the probability was $20 \%$ for a Neapolitan at the third, and $20 \%$ for a Neapolitan at the fifth position.

Procedure: In Experiments 1 and 2 [15], participants were only informed about the deviant instruments, not about the Neapolitan chords or the clusters. Participants were instructed to ignore the harmonies, to count the deviant instruments, and to report the number of deviant instruments when asked by pressing a response button. After an inquiry, the following chord sequences were from another key (resulting in seven sub-blocks from a different key). During Block 2 [16], subjects were playing a video-game under the instruction to ignore all acoustic stimuli.

Data analysis: Continuous EEG data from 25 scalp locations of the 10-20 system were referenced off-line to the algebraically mean of left and right mastoid electrodes. From the continuous raw EEGs, trials were averaged offline; the epoch-length was $1100 \mathrm{~ms}$, including a pre-stimulus baseline from -100 to $0 \mathrm{~ms}$ (with respect to the onset of a chord). ERPs elicited by appropriate (in-key) and inappropriate harmonies (clusters and Neapolitans cumulated) were averaged separately for the third and the fifth position, and separately for men and women. Data sets of the three experiments were pooled, the collapsing of the data sets is justified by the similarities in stimulation and paradigm: all experiments had (a) identical in-key chords, (b) identical timing, (c) in all experiments harmonically unexpected musical events were presented within a musical context with (d) similar probablilities at (e) identical positions within the chord sequences (third and fifth position). Note that the unexpected harmonies investigated in the mentioned experiments (clusters and Neapolitans) elicit both ERAN and N5, which are the subject of investigation in the present study.

For statistical analyses, two regions of interest (ROI) were computed [20]: a region of left frontal (F7, F3, FT7, FC3) and a region of right frontal electrodes (F4, F8, FC4, FT8). These ROIs correspond to the ROIs analyzed in the previous studies $[15,16]$. The computation of ROIs is a means to avoid loss of statistical power when ANOVAs are used to quantify potentials measured by adjacent electrodes [20]. Differences between mean amplitudes of these regions were tested by conducting repeated measurement ANOVAs. Global ANOVAs were conducted with the factors chord type (appropriate $\times$ inappropriate harmonies), lateralization (left $\times$ right hemisphere), and gender. Time windows for statistical 
analyses were chosen around the amplitude maxima of effects. In the time windows analyzed, effects had a stable scalp topography. After the statistical evaluation, ERPs were for presentation low-pass filtered at $10 \mathrm{~Hz}$ (41 points, FIR).

\section{RESULTS}

Figure 2 shows the ERPs elicited at the fifth position of the chord sequences, separately for males and females. The data indicate that the inappropriate harmonies elicited (in both males and females) an early as well as a late negativity. In both groups, the amplitude of the early negativity (the ERAN) was maximal around $200 \mathrm{~ms}$ after the onset of the chord, the late negativity (the N5) peaked around $550 \mathrm{~ms}$. The mean amplitudes of these effects did not differ between groups. Whereas the early negativity had a clear right hemispheric preponderance in males, it was bilateral in females (Figs. 2 and 3). This difference in scalp topography was not due to larger potentials over the right hemisphere of males, but due to smaller potentials in males over the left hemisphere.

These observations were supported by a statistical analysis of the scalp topographies. A global ANOVA for a time window from 130 to $280 \mathrm{~ms}$ revealed an effect of chord type $(\mathrm{F}(1,60)=58.49, p<0.0001)$, indicating that the effect of the inappropriate harmonies is significant), an interaction between factors chord type and hemisphere $(\mathrm{F}(1,60)=5.66$, $p<0.02)$, indicating that this effect was lateralized), and an interaction between factors chord type, lateralization, and gender $(\mathrm{F}(1,60)=4.24, p<0.05)$, indicating that the lateralization of the effect differed between males and females). No further two-way interactions were found. To validate the three-way interaction, the same analysis was performed after amplitude normalization [21]. In this analysis the interaction remained significant $(\mathrm{F}(1,60)=4.96, p<0.03)$. Consequently, it has to be concluded that the scalp topography of the early negative effect elicited by the harmonically inappropriate chords differed in its lateralization between genders. When data sets were analyzed separately for each experiment, the three-way interaction (chord type $\times$ lateralization $\times$ gender) was not clearly significant in any single experiment, but in two of the three experiments the estimated $p$-value was $<0.07$.

When analyzing the pooled data sets separately for males and females, data for males show a clear interaction between the factors chord type and hemisphere $(\mathrm{F}(1,30)=5.37, p<0.005)$, indicating that the effect was lateralized), whereas data for females show no interaction $(p>0.75)$.

Although the late negativity seems to be larger in females, and probably stronger lateralized in males, these differences were statistically not significant. An ANOVA for a time window from 450 to $600 \mathrm{~ms}$ revealed an effect of chord type $(\mathrm{F}(1,60)=28.37, \quad p<0.0001)$, but no two- or three-way interaction.

As already shown in the data from the single experiments $[15,16]$, early and late negativities elicited by the inappropriate harmonies were significantly smaller at the third position than at the fifth position, because the musical context was built up to a smaller degree in the middle of the sequence, leading to less specific expectancies (compared to
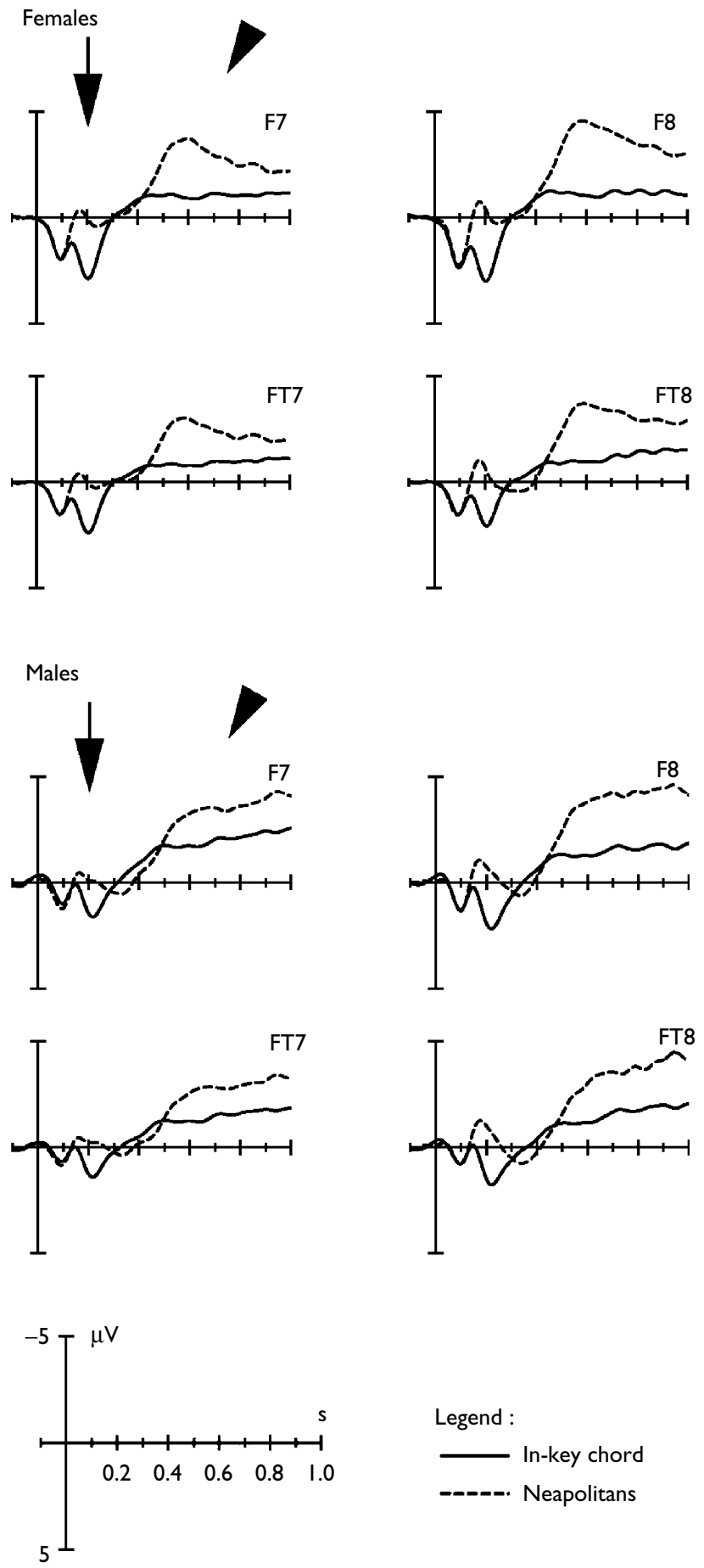

Legend :

In-key chord

---n-.. Neapolitans

Fig. 2. ERP waveforms of the electric potentials elicited at the fifth position of the chord sequences by appropriate (solid line) and inappropriate harmonies (Neapolitan chords and clusters, dashed line), averaged across females (top) and males (bottom). Electrodes of the left column (F7, FT7) represent left anterior leads, electrodes of the right colum (F8, FT8) represent right anterior leads. In both groups, the inappropriate harmonies elicited an early negativity (long arrow) which was followed by a late negativity (short arrow). The early negativity had a bilateral scalp distribution in females and was preponderant over the right hemisphere in males. Whereas the amplitude of the early negativity did not differ between genders over the right hemisphere, the potential was smaller over the left hemisphere in males. 


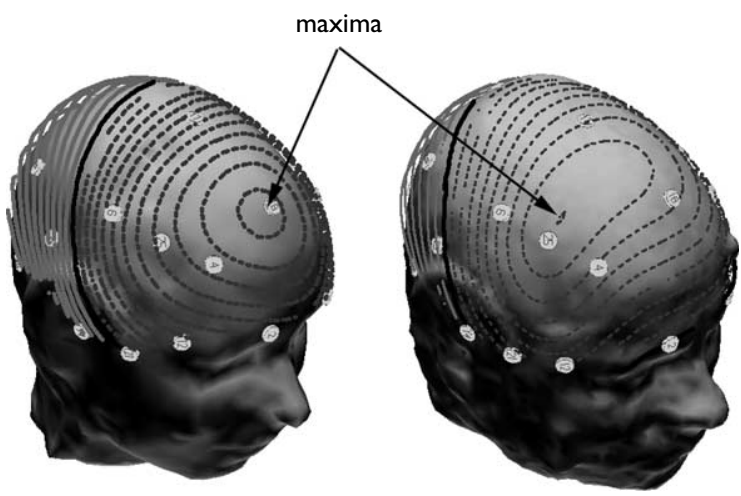

Fig. 3. Scalp distribution of the early effect of inappropriate harmonies, separately for females (left) and males (right). The figures show isopotential lines of the difference ERPs (appropriate subtracted from inappropriate harmonies) $200 \mathrm{~ms}$ after the onset of the stimuli. The thick line indicates the zero-potential line, the dashed lines anterior to the thick line indicate negative potential values, the solid lines posteriorly indicate positive values; the electrical potential difference displayed by two adjacent isopotential lines is $0.2 \mu \mathrm{V}$. The potential maps show that the early effect is lateralized to the right in males and bilateral in females (the arrows indicate the negative potential maxima of the effects).

at the end of the sequence, where a strong expectancy for the tonic chord was established) [15-17].

\section{DISCUSSION}

The present study reveals that the early negativity elicited by inappropriate harmonies presented within a musical context has a bilateral potential distribution over the scalp in females and a right-lateralized distribution in males. The early negativity has been taken to reflect a musical sound expectancy violation, the musical expectancies being generated during the perception of a preceding musical context, based on (implicit) knowledge of complex musical regularities [22-24] (the implicit knowledge being most presumably culture specific).

The system of musical regularities of major-minor tonal music is described by music theory (with respect to the succession of chord functions, the harmonic relationships between chords and keys, part writing, etc.) and may be denoted as a musical syntax [13-15]. The term musical syntax does not imply that it is a linguistic syntax in musical terms; rather it points to the fact that music is structured according to complex regularities (a feature reminiscent of language).

With respect to the differences in scalp topography between genders it is interesting to note that the ERAN has been suggested to be generated in the inferior frontolateral cortex (BA 44), which is in the left hemisphere part of Broca's area [14] (this observation was supported by a recent fMRI study [19]). The present results are not taken as evidence for a gender-specific modulation of the ERAN in BA44, as claims about generators cannot be made based on scalp topography alone. However, both functional [2-4] and anatomical $[25,26]$ gender differences have been reported for BA 44: in functional imaging studies, activations of language functions in this region were more strongly leftlateralized in males than in females (who showed more bilateral activations) [2-4]. Anatomical studies indicate that the cell density of BA 44 is lower in the right hemisphere in males, but not in females [25], and that Broca's area is proportionally larger in the female brain [26]. The functional and structural gender differences have been taken as evidence for a more symmetrical control of verbal functions in women and, correspondingly, to a more lateralized control in men. The gender differences reported in previous studies thus correspond with the present observation that the early music negativity is lateralized in males and bilateral in females, although with a hemispheric weighting different from that of language.

It is highly plausible that the observed gender difference is due to a gender-specific hemispheric weighting in the recruitment of neural ressources, e.g. a gender-wise difference in the strength or number of generators; this interpretation concurs with the mentioned results of previous functional imaging studies [2-4]. A gender-wise difference in the orientation of identical generators in each hemisphere is rather unlikely, because this would require gross anatomical gender differences in gyrification and sulcification (note that the present data were obtained with EEG). With this respect, the present data strongly suggest that females process music-syntactic information bilaterally, and males with a right-hemispheric predominance. Note, however, that the early negativity of males was also present over the left hemisphere, indicating that males did not exclusively employ the right hemisphere for the processing of the musical information.

Note that the present study, as well as previous studies [14-18], indicate some similarities and differences between the ERAN and the mismatch negativity (MMN) [27]. An important difference is that the amplitude of the ERAN depends on a regularity-based generation of harmonic expectancies for functionally appropriate chords [14,16,17], whereas the MMN is broadly assumed to reflect sensory memory operations [27]. In addition, the present study shows that the ERAN differs in its scalp distribution between males and females, which has (at least so far) not been reported for the MMN. However, the ERAN and the MMN also share a number of features [15-17], including the sensitivity to stimuli that do not match with a previously presented group of events. Because the early negativity was lateralized only in males, the term early right anterior negativity falls short as a descriptor for the effect in general. Given the similarities between ERAN and MMN, and the findings that the ERAN is sensitive to violations of complex musical regularities, the ERAN has previously been suggested to be understood as a music-syntactic MMN [18].

\section{CONCLUSION}

The present results indicate that relatively early electric brain activity elicited by inappropriate harmonies within a musical sequence is distributed bilaterally over the scalp in females, and lateralized to the right hemisphere in males. This finding reveals gender differences for the processing of musical information, indicating that gender differences in auditory information processing are not restricted to processes in the linguistic domain such as syntax, semantics, and phonology. Interestingly, because of the female brain's ability to process language bilaterally, it appears that (a) females can better compensate aphasic impairments 
[6], (b) girls have fewer language disorders than boys [8], and (c) females have facilitated language and verbal learning [28]. The gender difference observed in the present study might contribute to these effects, because the processing of musical elements of speech is an important aspect of speech perception and language acquisition $[29,30]$. Finally, on the basis of the present findings one should predict differential impairment in syntactic music processing between men and women suffering from lesions within the right inferior fronto-lateral cortex.

\section{REFERENCES}

1. Kimura D. Sex and Cognition. Cambridge: MIT Press; 1999.

2. Pugh K, Shaywitz B, Shaywitz S et al. Brain 119, 1221-1238 (1996).

3. Jaeger J, Lockwood A, Valin R et al. Neuroreport 9, 2803-2807 (1998).

4. Kansaku K, Yamaura A and Kitazawa S. Cerebr Cortex 10, 866-872 (2000).

5. Phillips M, Lowe M, Dzemidzic M et al. Radiology 220, 202-207 (2001).

6. McGlone J. Behav Brain Sci 3, 215-263 (1980).

7. Moulden J and Persinger M. Percept Mot Skills 90, 893-898 (2000).

8. Hier D. Bull Orton Soc 29, 74-83 (1979).

9. Platel H, Price C, Baron J et al. Brain 120, 229-243 (1997).

10. Liegeois-Chauvel C, Peretz I, Babaie M et al. Brain 121, 1853-1867 (1998).
11. Peretz I, Kolinsky R, Tramo M et al. Brain 117, 1283-1301 (1994).

12. Zatorre R, Evans A and Meyer E. J Neurosci 14, 1908-1919 (1994).

13. Patel AD, Gibson E, Ratner J et al. J Cogn Neurosci 10, 717-733 (1998).

14. Maess B, Koelsch S, Gunter T et al. Nature Neurosci 4, 540-545 (2001).

15. Koelsch S, Gunter T, Friederici AD et al. J Cogn Neurosci 12, 520-541 (2000).

16. Koelsch S, Gunter T, Schröger E et al. Neuroreport 12, 1385-1389 (2001)

17. Koelsch S, Schröger E and Gunter T. Psychophysiology 39, 1-11 (2002).

18. Koelsch S, Schmidt B and Kansok J. Psychophysiology 39, 657-663 (2002).

19. Koelsch S, Gunter T, von Cramon DY et al. Neuroimage 17, 956-966 (2002).

20. Oken B and Chiappa K. Ann Neurol 19, $493-494$ (1986).

21. McCarthy G and Wood C. Electroencephalogr Clin Neurophysiol 69, 218-233 (1985).

22. Krumhansl C and Kessler E. Psychol Rev 89, 334-368 (1982).

23. Bharucha J and Krumhansl C. Cognition 13, 63-102 (1983).

24. Tillmann B, Bharucha J and Bigand E. Psychol Rev 107, 885-913 (2000).

25. Amunts K, Schleicher A, Burgel U et al. J Comp Neurol 412, 319-341 (1999).

26. Harasty J, Double K, Halliday G et al. Arch Neur 54, 171-176 (1997).

27. Schröger E. Behav Res Meth Instr Comp 30, 131-145 (1998).

28. Kramer JH, Delis DC, Kaplan E et al. Neuropsychology 11, 577-584 (1997).

29. Steinhauer K, Alter K and Friederici AD. Nature Neurosci 2, 191-196 (1999).

30. Papoušek H. In: Sloboda J and Deliege I (eds). Musical Beginnings. Oxford: Oxford University Press; 1996. 